\title{
Sarcomatous Carcinoma of the Orbit: A Case Report and Review of the Literature
}

\author{
Ninan Mathew ${ }^{a} \quad$ Mathen Mathew ${ }^{b} \quad J$ Jarrah ${ }^{c}$ \\ ${ }^{a}$ Oral and Maxillofacial Surgery, Queensland Health, The University of Queensland, \\ Herston, QId., ' General Surgery, Monash Health, Clayton, Vic., and 'Queensland Health, \\ South Brisbane, QId., Australia
}

\section{Key Words}

Orbital · Sarcomatoid · Squamous · Carcinoma $\cdot$ Head and neck $\cdot$ Spindle cell tumours

\begin{abstract}
Sarcomatous carcinomas (SCs) are rare tumours that contain malignant cells with epithelial and mesenchymal characteristics. SC rarely presents in the head and neck, and occurs even less often in the orbit. Only 8 cases of SCs located in the orbit or affecting the globe function have been described in the English literature. Here, we report a case of SC affecting the right orbit. SC is associated with a poor prognosis and advanced disease at presentation. Diagnosis is difficult, as histology often fails to definitively identify SC, necessitating a wide panel of molecular/immunological markers. Treatment options are generally aggressive but risky, and frequently yield poor results. Due to the rarity of SC, there has been little focus on the development of improved treatment options.

(c) 2015 S. Karger AG, Basel
\end{abstract}

\section{Introduction}

Sarcomatoid or sarcomatous carcinoma (SC) is a rare tumour type that contains epithelial ('carcinoma') and mesenchymal ('sarcoma') malignant cells. We present a review of the literature and a case study of a patient with orbital SC. 
Mathew et al.: Sarcomatous Carcinoma of the Orbit: A Case Report and Review of the Literature

\section{Case Report}

A 71-year-old woman with type 2 diabetes mellitus, atrial fibrillation, congestive heart failure, chronic renal failure (oxalate crystal nephropathy), pulmonary hypertension, rheumatic heart disease (with mitral valve replacement), and multiple basal and squamous cell carcinomas affecting the face, back, and arms was evaluated for symptoms of floaters and subsequent progressive loss of vision and painful proptosis $(5 \mathrm{~mm}$, as measured by Hertel exophthalmometer) in the right eye. Upon examination, abduction and the ability to gaze upward on the right were restricted in the right eye. Her visual acuity was restricted to light perception only in the right eye (6/18 in the left eye). A relative afferent pupillary defect and global external ophthalmoplegia were also present on the right. A fundus exam revealed a central retinal vein occlusion. A diminished corneal sensation also was observed.

A CT without contrast enhancement (due to significant renal impairment) demonstrated an ill-defined soft tissue lesion in the right superolateral margin of the right orbit; the lesion encased the right superior rectus and lateral rectus muscles. An MRI revealed a right extraconal lesion involving the right lateral rectus and superior rectus muscles, resulting in proptosis (fig. 1). The initial biopsy was benign, showing mainly a dense collagenous tissue. Gram stain, culture, and polymerase chain reaction were negative for infection.

Following the gradual and complete deterioration of her visual acuity over a 6-month period, a second review was performed. The CT revealed significant disease progression. In addition, positron emission tomography revealed low fluorodeoxyglucose uptake in the diffuse infiltrative right retro-orbital mass with a standardised uptake value of 2.6.

The right eye was enucleated, and multiple biopsies from the right orbit revealed sarcomatous carcinoma.

\section{Pathologic Findings}

A gross examination of the tumour revealed that the right eye had a diameter of $18 \mathrm{~mm}$ with an indurated and ragged area on the surface of the posterior aspect of the globe; the tumour was located near the optic nerve margin. Biopsies appeared as pink and purple fragments measuring on average $25 \times 14 \mathrm{~mm}$.

A microscopic examination revealed a spindle cell proliferation involving the optic nerve root extrinsic to the eye with an extensive invasion of the sclera. The tumour was composed of malignant spindle cells in a collagenous stroma with moderate nuclear pleomorphism (fig. 2). A retinal detachment with focal tumour extension towards the inner aspect of the eye (through almost the entire thickness of the sclera) was observed.

Biopsy fragments demonstrated malignant spindle cells with pleomorphic nuclei, eosinophilic cytoplasm, and some cohesive areas with epithelioid tumour cells; other areas revealed tumour cells with bright eosinophilic cytoplasm, suggesting a squamous differentiation. Furthermore, immunohistochemistry (IHC) analyses for smooth muscle actin and muscle-specific actin were moderately positive (consistent with sarcomatoid differentiation). IHC analyses also were positive for AE1/AE3, high-molecular-weight keratin (HMWK) 34BE12, and vimentin (fig. 3), supporting the diagnosis of SC.

\section{Management}

In addition to the initial enucleation and biopsies, the patient received localised radiotherapy (60 Gy in 30 fractions). The patient opted not to proceed with more aggressive surgical options or chemotherapy due to comorbid disease, general frailty, and risks of complications. Unfortunately, an infection occurred soon after radiotherapy and was treated with regular antibiotics. The patient also developed acute renal failure, which required dialysis. 
Mathew et al.: Sarcomatous Carcinoma of the Orbit: A Case Report and Review of the Literature

Grade 2 erythema on the irradiated skin was treated with regular moisturisers and 1\% hydrocortisone. A subsequent MRI showed stable disease. All details of this case were reproduced after obtaining consent from the patient.

\section{Discussion}

\section{Epidemiology}

There exist sparse data on the global incidence of SC. The main focus is on SC in pulmonary ( $1 \%$ of all lung malignancies) and urinary $(0.11 \%$ of all bladder malignancies) systems [1-3]; there is no information about the incidence of SC in the eye [4]. Our review of the literature and Prakalapakorn et al. [5] identified 6 cases of orbital carcinosarcoma, 11 cases of carcinosarcoma of the paranasal sinuses, and 1 case of sinonasal teratocarcinosarcoma. Our patient represented 1 of 9 cases in the literature in which SC affected the orbit and globe function.

\section{Aetiology}

Tobacco (smoked/chewed), alcohol, and radiation exposure are probable inducers of SC [6-8]. Previous hypotheses, such as the 'collision tumour theory', have suggested that SC is derived from 2 distinct cell lineages [3]. However, Kashiwabara et al. [9] provided molecular evidence of a monoclonal origin for a case of oesophageal SC. More recent studies of renal, pancreatic, bladder, and prostatic SC support a monoclonal epithelial cell origin with subsequent reprogramming of descendant cells, which results in the formation of mesenchymal cells $[3,10,11]$. Our patient had a history of multiple facial basal and squamous cell carcinomas, which may be indicative of the tumour origin.

\section{Diagnosis}

Early diagnosis is critical because SC is a particularly aggressive tumour type. Viswanathan et al. [11] developed an algorithm for the diagnosis of SC (online suppl. fig. 1; see www.karger.com/doi/10.1159/000431282), which is complex because of histopathological features that overlap with other spindle cell tumours. In the present case, this algorithm positively diagnosed SC. The histopathology revealed a spindle cell neoplasm with epithelioid and sarcomatoid differentiation, which was supported by positive IHC results for AE1/AE3, HMWK 34BE12, and vimentin and negative staining for CD117, melanA, and S100.

\section{Treatment}

The treatment for SC is generally aggressive, including a full resection with radiotherapy and less often chemotherapy [7]. Due to the paucity of cases and literature, there is no widely accepted protocol for the treatment of SC in any tissue type [8]. Prakalapakorn et al. [5] reported successful surgical resection in 1 patient with orbital carcinosarcoma with no recurrence reported 10 months after surgery. However, death occurred 13 months after tumour debulking in another patient. The remaining 4 cases of orbital carcinosarcoma were treated with an anterior orbitotomy with an unknown outcome, palliative radiation for bone pain with 1 month of survival, and 2 cases for which treatment and mortality data were not provided, respectively. In 11 cases of carcinosarcoma of the paranasal sinuses [5], 7 were treated with some combination of surgery, radiation, and/or chemotherapy with survival ranging from a few months to 1 year after treatment. The single case of sinonasal teratocarcinosarcoma was not treated, and only a 1-month survival was reported. In the case reported here, due to multiple comorbidities, general frailty, poor prognosis despite treatment, and 
Mathew et al.: Sarcomatous Carcinoma of the Orbit: A Case Report and Review of the Literature

a desire for quality of life, no further surgery was attempted, and chemotherapy was declined. Subsequent management included localised radiotherapy and observation.

\section{Prognosis}

The progression of SC has been reported to be rapid and characterised by recurrences and metastases after local therapy $[11,15]$. The overall prognosis for SC is poor, as patients usually present with late-stage disease, frequent nodal involvement, and distant metastatic spread [8]. Advanced patient age, female sex, and the evidence of distant metastases adversely affect prognosis [14]. Inadequate response to chemo/immunotherapy also is associated with poor survival rates [14]. However, mitotic count, the degree of pleomorphism, and cellularity do not appear to correlate with the prognosis [14].

\section{Conclusion}

Sarcomatoid carcinoma of the orbit is a rare tumour with an aggressive course and poor prognosis. Treatment options are usually aggressive, including surgical resection, targeted radiotherapy, and, to a lesser extent, chemotherapy. In the cases reported thus far in the literature, the prognosis remains poor. Due to the paucity of the literature and the poor prognosis of the disease, the development of effective treatments has been slow to progress [3].

\section{Acknowledgement}

Special thanks go out to Dr. John Dooley for providing the microphotographs of the histological and IHC slides.

\section{Statement of Ethics}

All details of this case were reproduced after obtaining consent from the patient and the patient's next of kin. Any treatments undertaken by the patient were without deviation from accepted standards of care. This report represents a retrospective accounting alone.

\section{Disclosure Statement}

The authors declare no actual or potential conflicts of interest.

\section{References}

1 Franks TJ, Galvin JR: Sarcomatoid carcinoma of the lung: histologic criteria and common lesions in the differential diagnosis. Arch Pathol Lab Med 2010;134:49-54.

2 Kumar M, Goyal S, Bahl A, Das P, Sharma DN, Ray R, Rath GK: Sarcomatoid carcinoma of the maxillary sinus: a rare head and neck tumor. J Cancer Res Ther 2008;4:131-133.

-3 Wang J, Wang FW, Lagrange CA, Hemstreet Iii GPH, Kessinger A: Clinical features of sarcomatoid carcinoma (carcinosarcoma) of the urinary bladder: analysis of 221 cases. Sarcoma 2010;2010:1-7.

-4 Tang W, Hei Y, Xiao L: Recurrent orbital space-occupying lesions: a clinicopathologic study of 253 cases. Chin J Cancer Res 2013;25:423-429. 
Mathew et al:: Sarcomatous Carcinoma of the Orbit: A Case Report and Review of the Literature

5 Prakalapakorn SG, Bernardino CR, Auclair PL, Grossniklaus HE: Carcinosarcoma of the orbit: report of two cases and review of the literature. Ophthalmology 2008;115:2065-2070.

6 Italiano A, Cortot AB, Ilie M, Martel-Planche G, Fabas T, Pop D, Mouroux J, Hofman P, Pedeutour F: EGFR and KRAS status of primary sarcomatoid carcinomas of the lung: implications for anti-EGFR treatment of a rare lung malignancy. Int J Cancer 2009;125:2479-2482.

-7 Perret L, Chaubert P, Hessler D, Guillou L: Primary heterologous carcinosarcoma (metaplastic carcinoma) of the urinary bladder: a clinicopathologic, immunohistochemical, and ultrastructural analysis of eight cases and a review of the literature. Cancer 1998;82:1535-1549.

8 Dundore PA, Cheville JC, Nascimento AG, Farrow GM, Bostwick DG: Carcinosarcoma of the prostate. Report of 21 cases. Cancer 1995;76:1035-1042.

-9 Kashiwabara K, Sano T, Oyama T, Najahima T, Makita F, Hashimoto N, Iwanami K, Kawashima O, Matsumoto T, Matsuzaki Y: A case of esophageal sarcomatoid carcinoma with molecular evidence of a monoclonal origin. Pathol Res Pract 2001;197:41-46.

$\checkmark 10$ Jones TD, Eble JN, Wang M, Maclennan GT, Jain S, Cheng L: Clonal divergence and genetic heterogeneity in clear cell renal cell carcinomas with sarcomatoid transformation. Cancer 2005;104:1195-1203.

11 Viswanathan S, Rahman K, Pallavi S, Sachin J, Patil A, Chaturvedi P, D’Cruz A, Agarwal J, Kane SV: Sarcomatoid (spindle cell) carcinoma of the head and neck mucosal region: a clinicopathologic review of 103 cases from a tertiary referral cancer centre. Head Neck Pathol 2010;4:265-275.

-12 Bahrami A, Truong LD, Ro JY: Undifferentiated tumor: true identity by immunohistochemistry. Arch Pathol Lab Med 2008;132:326-348.

13 Moon JK, Kim AY, Chang DS, Park KY: Carcinosarcoma of the maxillary sinus. Clin Exp Otorhinolaryngol 2013;6:114-116.

14 Cangiano T, Liao J, Naitoh J, Dorey F, Figlin R, Belldegrun A: Sarcomatoid renal cell carcinoma: biologic behavior, prognosis, and response to combined surgical resection and immunotherapy. J Clin Oncol 1999;17:523-528.

15 Giunchi F, Vasuri F, Baldin P, Rosini F, Corti B, D’Errico-Grigioni A: Primary liver sarcomatous carcinoma: report of two cases and review of the literature. Pathol Res Pract 2013;209:249-254.
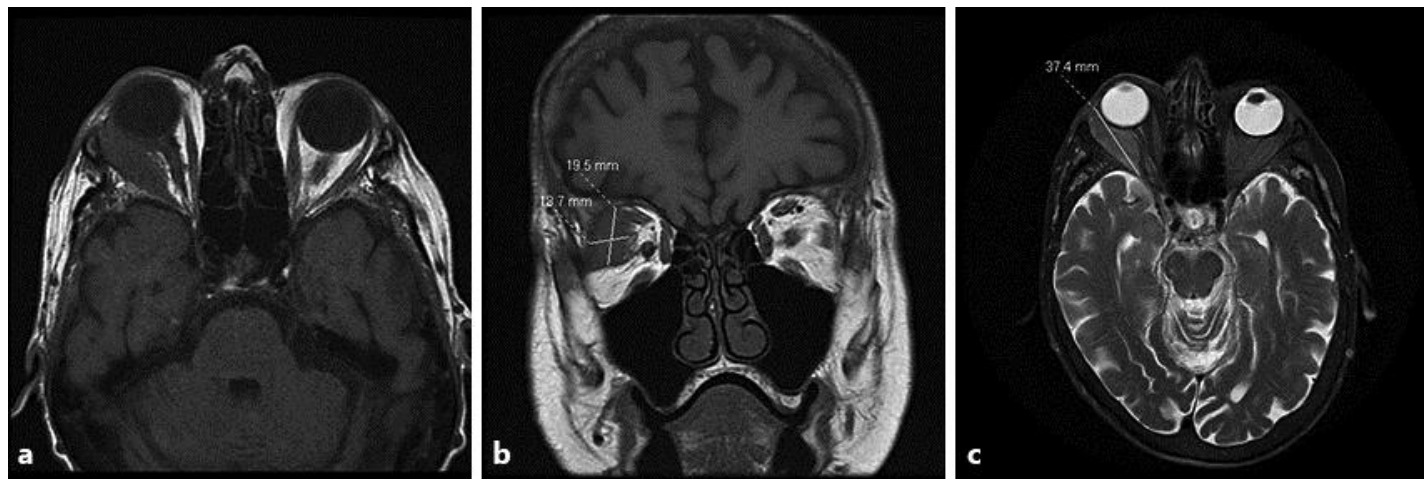

Fig. 1. a MRI: T1-weighted axial views of the head. b T1-weighted coronal views of the head. c T2-weighted axial views of the head. 


\section{Case Reports in \\ Ophthalmology}
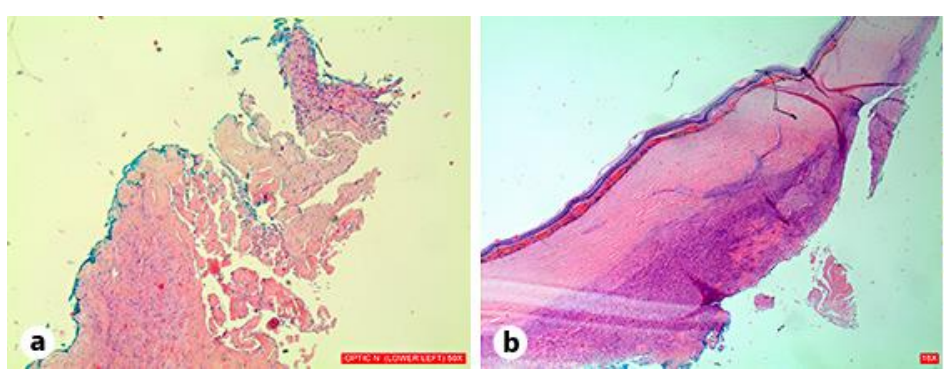

\begin{tabular}{l|l}
\hline \multicolumn{2}{l}{ Case Rep Ophthalmol 2015;6:180-185 } \\
\hline DOI: 10.1159/000431282 & $\begin{array}{l}\text { ○ 2015 S. Karger AG, Basel } \\
\text { www.karger.com/cop }\end{array}$ \\
\hline
\end{tabular}

Mathew et al.: Sarcomatous Carcinoma of the Orbit: A Case Report and Review of the Literature

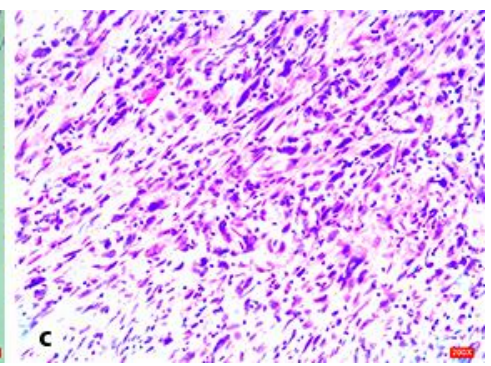

Fig. 2. a Histology: HE staining of the optic nerve at $\times 50$ magnification. b HE. $\times 16$ (resection specimen). c HE. $\times 200$ (resection specimen).
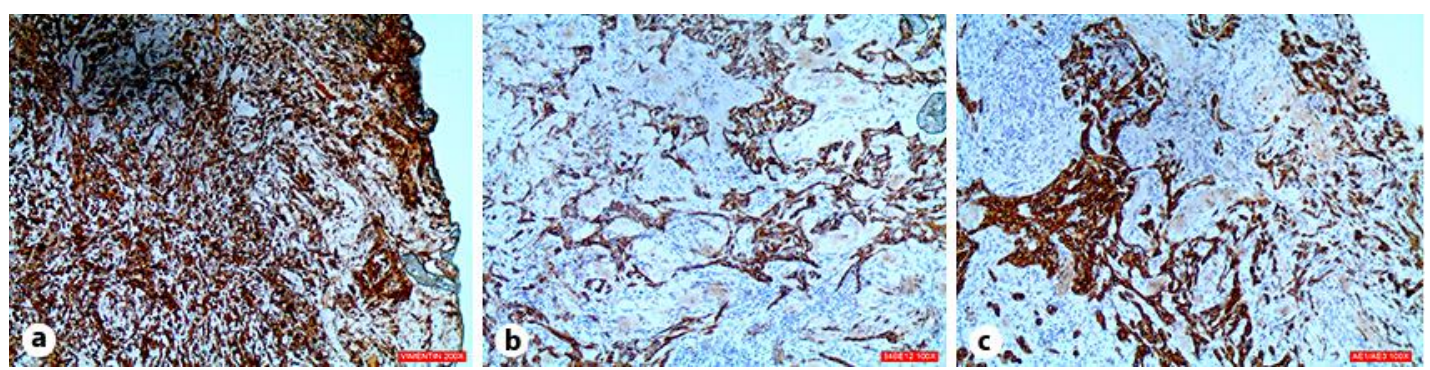

Fig. 3. a Immunohistochemistry: vimentin-positive staining of resection specimen at $\times 200$ magnification. b 34BE12-positive staining of resection specimen at $\times 100$ magnification. c AE1/AE3-positive staining of resection specimen at $\times 100$ magnification. 